\title{
LOCAL INTERPOLATION WITH OPTIMAL POLYNOMIAL EXACTNESS IN REFINEMENT SPACES
}

\author{
JOHAN DE VILLIERS AND MPFARELENI REJOYCE GAVHI
}

\begin{abstract}
A constructive existence result for a class of polynomial identities is established and applied in two related contexts. First, an algorithm is developed for the explicit construction of a sequence of local interpolation operators mapping the space of continuous functions on the real line into the nested sequence of refinement spaces generated by the shifts of a given refinable function, and where optimal polynomial exactness, as governed by the order of the sum-rule condition satisfied by the corresponding refinement sequence, is achieved. The above algorithm requires as input only the values at the integers of the refinable function, and we proceed, secondly, to derive sufficient conditions for the existence of a refinable function with prescribed values at the integers. As our main examples, we consider the cardinal $B$-spline case, as well as refinable functions with normalized binomial coefficient values at the integers.
\end{abstract}

\section{INTRODUCTION}

We write $\ell(\mathbb{Z})$ for the space of all bi-infinite real-valued sequences $\left\{c_{j}\right\}=\left\{c_{j}\right.$ : $j \in \mathbb{Z}\}$, and we denote by $\ell_{0}(\mathbb{Z})$ the subspace of $\ell(\mathbb{Z})$ consisting of sequences $\left\{c_{j}\right\}$ such that $\left\{j: c_{j} \neq 0\right\}$ is a finite set, in which case we call $\left\{c_{j}\right\}$ a finitely supported sequence. We use the standard symbol $C(\mathbb{R})$ for the space of continuous functions $f: \mathbb{R} \rightarrow \mathbb{R}$, and we write $C_{0}(\mathbb{R})$ for the subspace of $C(\mathbb{R})$ consisting of functions which vanish identically outside some bounded interval, in which case we say that $f$ is a compactly supported function. For any non-negative integer $k$, the symbol $\pi_{k}$ will denote the space of polynomials of degree at most $k$, and $C^{k}(\mathbb{R})$ is defined as the subspace of functions $f \in C(\mathbb{R})$ such that $f^{(k)} \in C(\mathbb{R})$, so that $C^{0}(\mathbb{R})=C(\mathbb{R})$. Also, $C_{0}^{k}(\mathbb{R}):=C^{k}(\mathbb{R}) \cap C_{0}(\mathbb{R})$.

In this paper, we say that $\phi$ is a refinable function with refinement sequence $\left\{p_{j}\right\}$ if $\phi \in C_{0}(\mathbb{R}),\left\{p_{j}\right\} \in \ell_{0}(\mathbb{Z})$, and the refinement equation

$$
\phi(x)=\sum_{j} p_{j} \phi(2 x-j), \quad x \in \mathbb{R},
$$

where $\sum_{j}:=\sum_{j \in \mathbb{Z}}$, is satisfied. Refinable functions play an important role in the mathematical analysis of both wavelets and subdivision (see e.g., [1, [2, [3], 6], [7], 10]).

Received by the editor May 17, 2013 and, in revised form, August 22, 2014. 2010 Mathematics Subject Classification. Primary 65D05; Secondary 65D07. 
For any refinable function $\phi$, it follows from (1.1) that the corresponding refinement space sequence

$$
S_{r}:=\left\{\sum_{j} c_{j} \phi\left(2^{r} \cdot-j\right):\left\{c_{j}\right\} \in \ell(\mathbb{Z})\right\}, \quad r \in \mathbb{Z},
$$

satisfies the nesting property

$$
S_{r} \subset S_{r+1}, \quad r \in \mathbb{Z} .
$$

A sequence $\left\{a_{j}\right\} \in \ell_{0}(\mathbb{Z})$ is said to satisfy the sum-rule condition of order $m \in \mathbb{N}$ if it holds that

$$
\left.\begin{array}{c}
\beta_{\ell}:=\sum_{j}(2 j)^{\ell} a_{2 j}=\sum_{j}(2 j+1)^{\ell} a_{2 j+1}, \quad \ell=0, \ldots, m-1 ; \\
\text { with } \quad \beta_{0}=1,
\end{array}\right\}
$$

and where $m$ is the largest integer for which (1.4) holds. The second line of (1.4), namely

$$
\sum_{j} a_{2 j}=\sum_{j} a_{2 j+1}=1,
$$

is referred to as the sum rule.

As proved in [2, Theorem 5.3.1], a sequence $\left\{a_{j}\right\} \in \ell_{0}(\mathbb{Z})$ satisfies the sum-rule condition of order $m \in \mathbb{N}$ if and only if the corresponding Laurent polynomial symbol

$$
A(z):=\frac{1}{2} \sum_{j} a_{j} z^{j}
$$

satisfies the formulation

$$
A(z)=\left(\frac{1+z}{2}\right)^{m} \tilde{A}(z)
$$

for some Laurent polynomial $\tilde{A}$ such that

$$
\tilde{A}(1)=1 ; \quad \tilde{A}(-1) \neq 0 .
$$

Let $\phi$ denote a refinable function with refinement sequence $\left\{p_{j}\right\}$ satisfying the sum-rule condition of order $m \in \mathbb{N}$, as will be assumed henceforth. Then (see [2, Corollary 5.22]), the polynomial containment property $\pi_{m-1} \subset S_{r}, r \in \mathbb{Z}$, is satisfied, with also $\pi_{m}$ not contained in $S_{r}$, for any $r \in \mathbb{Z}$. Our main objective in this paper is, for any $r \in \mathbb{Z}$, the explicit construction of an approximation operator $\mathcal{L}_{r}: C(\mathbb{R}) \rightarrow S_{r}$ such that:

(A) $\mathcal{L}_{r}$ is local in the sense that, for any $f \in C(\mathbb{R}), r \in \mathbb{Z}$ and $x \in \mathbb{R}$, the value $\left(\mathcal{L}_{r} f\right)(x)$ depends on the values of $f(y)$ only for $y \in J_{r}:=\left\{\frac{j}{2^{r-1}}: j=j_{0}, \ldots, j_{1}\right\}$, with $j_{0}$ and $j_{1}$ denoting integers such that $x \in\left[\frac{j_{0}}{2^{r-1}}, \frac{j_{1}}{2^{r-1}}\right]$, and where the difference $j_{1}-j_{0}$ is a positive integer which is independent of $x$ and $r$;

(B) the interpolatory conditions

$$
\left(\mathcal{L}_{r} f\right)\left(\frac{j}{2^{r-1}}\right)=f\left(\frac{j}{2^{r-1}}\right), \quad j \in \mathbb{Z},
$$

are satisfied for each $f \in C(\mathbb{R})$; 
(C) the optimal polynomial exactness property

$$
\mathcal{L}_{r} f=f, \quad f \in \pi_{m-1},
$$

is satisfied.

Such a local interpolation operator $\mathcal{L}_{r}$ with optimal polynomial exactness could prove useful, for example, in the first step of any wavelet decomposition algorithm based on the refinable function $\phi$, in which a given signal $f$ needs to be efficiently mapped into a refinement space $S_{r}$ of sufficiently high resolution level $r$.

Our construction of $\mathcal{L}_{r}$ will be based on the sequence $\left\{y_{j}\right\} \in \ell_{0}(\mathbb{Z})$ defined by

$$
y_{j}:=\phi(j), \quad j \in \mathbb{Z} .
$$

Since the refinement sequence $\left\{p_{j}\right\}$ satisfies the sum rule, we may assume without loss of generality (see [2, Theorem 4.3.2 and Lemma 4.4.1]) that

$$
\sum_{j} \phi(x-j)=1, \quad x \in \mathbb{R}
$$

Let us furthermore assume that, for some integer $\nu \geq 2$, the finitely supported refinement sequence $\left\{p_{j}\right\}$ has support interval

$$
\operatorname{supp}\left\{p_{j}\right\}=\left.[0, \nu]\right|_{\mathbb{Z}},
$$

that is, $\min \left\{j: p_{j} \neq 0\right\}=0 ; \max \left\{j: p_{j} \neq 0\right\}=\nu$. According to a standard result (see e.g., [2, Theorem 2.1.1]), the convex hull of the support of $\phi$ is then given by

$$
\operatorname{supp}^{c} \phi=[0, \nu],
$$

that is, $\inf \{x: \phi(x) \neq 0\}=0 ; \sup \{x: \phi(x) \neq 0\}=\nu$. It follows from (1.11) and (1.14) that

$$
\left.\operatorname{supp}\left\{y_{j}\right\} \subset[1, \nu-1]\right|_{\mathbb{Z}},
$$

and thus also, from (1.11) and (1.12),

$$
\sum_{j} y_{j}=\sum_{j=1}^{\nu-1} y_{j}=1 .
$$

Now observe from (1.1) and (1.11) that

$$
y_{j}=\sum_{k} p_{k} y_{2 j-k}=\sum_{k} p_{2 j-k} y_{k}, \quad j \in \mathbb{Z},
$$

which, together with (1.15), yields

$$
\sum_{k=1}^{\nu-1} p_{2 j-k} y_{k}=y_{j}, \quad j=1, \ldots, \nu-1,
$$

or, in equivalent matrix-vector form,

$$
\mathcal{P} \mathbf{y}=\mathbf{y}, \quad \text { with }[\mathcal{P}]_{j, k=1, \ldots, \nu-1}:=p_{2 j-k} ; \quad \mathbf{y}:=\left[y_{1}, \ldots, y_{\nu-1}\right]^{T} .
$$

Hence the matrix $\mathcal{P}$ has the eigenvalue 1 , and, provided that 1 is a simple eigenvalue of $\mathcal{P}$, the vector $\mathbf{y}$ may be calculated as the corresponding eigenvector satisfying the condition (1.16) and thereby completing the calculation of the sequence $\left\{y_{j}\right\}$.

Let the polynomial $Y \in \pi_{\nu-2}$ be defined by

$$
Y(z):=\sum_{j} y_{j+1} z^{j}=\sum_{j=0}^{\nu-2} y_{j+1} z^{j} .
$$


In Section 2, we characterise, in Theorem 1, and in terms of a polynomial identity involving $Y$, a class of local interpolation operators $\mathcal{L}_{r}$, as in (A) and (B). In addition, we observe that if the interpolation point sequence $\left\{\frac{j}{2^{r-1}}: j \in \mathbb{Z}\right\}$ in (1.9) were to be replaced by the finer sequence $\left\{\frac{j}{2^{r}}: j \in \mathbb{Z}\right\}$, then (A) and (B) are compatible only in the special case where the sequence $\left\{y_{j}\right\}$ in (1.11) has precisely one non-zero element.

We proceed in Section 3 to derive, in Theorem 2, a sufficient condition on the polynomial identity of Section 2 for the approximation operator $\mathcal{L}_{r}$ to also satisfy the optimal polynomial exactness property (1.10) in (C). As an extension of the result [2, Theorem 7.1.1], we then establish, in Section 4, a constructive existence theory in Theorem 3 for a general class of polynomial identities, a special case of which is subsequently applied in Theorem 4 of Section 5 to deduce the existence of, and obtain an explicit construction procedure for, as formulated in the subsequent Algorithm 1, an approximation operator $\mathcal{L}_{r}: C(\mathbb{R}) \rightarrow S_{r}$ satisfying the properties (A), (B) and (C). As an example, the specific choice $\phi=N_{m}$, the $m$-th order cardinal $B$-spline, is then considered, thereby yielding an efficient algorithm for the construction of the uniform knot case of a class of local spline interpolation operators studied for arbitrary spline knots in [4, 5] and [9].

Up to Section 5, the sequence $\left\{y_{j}\right\}$ in (1.11) is obtained from a given refinable function $\phi$ and its refinement sequence $\left\{p_{j}\right\}$. We proceed in Section 6 to investigate the following inverse problem: For a given sequence $\left\{y_{j}\right\} \in \ell_{0}(\mathbb{Z})$, investigate the existence of a refinable function $\phi$ satisfying the condition

$$
\phi(j)=y_{j}, \quad j \in \mathbb{Z},
$$

and thereby further developing the work in [8] (see also [11]) on this topic. By relying on the concept of subdivision convergence, as well as its relationship with positive refinement sequences, and by applying a further special case of the polynomial identity theory of Section 4, we establish in Theorems 5, 6 and 7, subject to some constraints on the sequence $\left\{y_{j}\right\}$, a constructive existence proof, as formulated in the subsequent Algorithm 2, for a refinement sequence $\left\{p_{j}\right\}$ satisfying the sum-rule condition of at least 2 and with corresponding refinable function $\phi$ satisfying (1.20). Finally, in Section 7 and following [8, Section 3], we consider the special case of Section 6 , where, in (1.20), $\left\{y_{j}\right\}$ is a sequence of normalized binomial coefficients for which the explicit construction procedure of Section 5 is then shown, in Theorems 8 and 9 , to yield an approximation operator $\mathcal{L}_{r}$ of particularly simple form.

\section{A LAURENT POLYNOMIAL IDENTITY FOR LOCAL INTERPOLATION}

We investigate the existence of a sequence $\left\{w_{j}\right\} \in \ell_{0}(\mathbb{Z})$ such that the compactly supported function

$$
w(x):=\sum_{j} w_{j} \phi(x-j)
$$

has the interpolation property

$$
w(2 j)=\delta_{j}, \quad j \in \mathbb{Z},
$$

with $\left\{\delta_{j}\right\}$ denoting the Kronecker delta sequence, that is,

$$
\delta_{j}:= \begin{cases}1, & j=0, \\ 0, & j \in \mathbb{Z} \backslash\{0\} .\end{cases}
$$


By recalling also the definition (1.2) of the refinement space sequence $\left\{S_{r}: r \in \mathbb{Z}\right\}$, we observe that if $w$ is given by (2.1), with the sequence $\left\{w_{j}\right\} \in \ell_{0}(\mathbb{Z})$ chosen such that (2.2) is satisfied, then, for any $r \in \mathbb{Z}$, the approximation operator $\mathcal{L}_{r}: C(\mathbb{R}) \rightarrow$ $S_{r}$, as given by

$$
\begin{array}{r}
\left(\mathcal{L}_{r} f\right)(x):=\sum_{j} f\left(\frac{j}{2^{r-1}}\right) w\left(2^{r} x-2 j\right)=\sum_{j}\left[\sum_{k} w_{j-2 k} f\left(\frac{k}{2^{r-1}}\right)\right] \begin{array}{r}
\phi\left(2^{r} x-j\right), \\
f \in C(\mathbb{R}),
\end{array}
\end{array}
$$

is a local interpolation operator, as in $(\mathrm{A})$ and $(\mathrm{B})$ of Section 1.

For any sequence $\left\{w_{j}\right\} \in \ell_{0}(\mathbb{Z})$, define the Laurent polynomial

$$
W(z):=\frac{1}{2} \sum_{j} w_{j} z^{j}
$$

The following characterisation result holds.

Theorem 1. Suppose $\phi \in C_{0}(\mathbb{R})$ is such that the support property (1.14) is satisfied for an integer $\nu \geq 2$, and let the polynomial $Y \in \pi_{\nu-2}$ be defined by (1.19), (1.11). Then $\left\{w_{j}\right\} \in \ell_{0}(\mathbb{Z})$ is a sequence such that the compactly supported function $w$, as defined by (2.1), satisfies the interpolation property (2.2) if and only if the Laurent polynomial $W$, as given by (2.5), satisfies the identity

$$
Y(z) W(z)-Y(-z) W(-z)=z^{-1}, \quad z \in \mathbb{C} \backslash\{0\} .
$$

Proof. First observe from (2.1) that a sequence $\left\{w_{j}\right\} \in \ell_{0}(\mathbb{Z})$ is such that the function $w$ satisfies (2.2) if and only if, for any $k \in \mathbb{Z}$,

$$
\delta_{k}=\sum_{j} w_{j} \phi(2 k-j)=\sum_{j} w_{2 k-j} \phi(j)=\sum_{j} y_{j} w_{2 k-j},
$$

after having recalled also the definition (1.11).

Now use the definitions (1.19) and (2.5) to deduce that (2.7) holds, for any $k \in \mathbb{Z}$, if and only if, for $z \in \mathbb{C} \backslash\{0\}$,

$$
\begin{aligned}
1 & =\sum_{k} \delta_{k} z^{k}=\sum_{k}\left[\sum_{j} y_{2 j} w_{2 k-2 j}\right] z^{2 k}+\sum_{k}\left[\sum_{j} y_{2 j+1} w_{2 k-2 j-1}\right] z^{2 k} \\
& =z\left\{\left[\sum_{j} y_{2 j} z^{2 j-1}\right]\left[\sum_{k} w_{2 k} z^{2 k}\right]+\left[\sum_{j} y_{2 j+1} z^{2 j}\right]\left[\sum_{k} w_{2 k+1} z^{2 k+1}\right]\right\} \\
& =2 z\left\{\left[\frac{Y(z)-Y(-z)}{2}\right]\left[\frac{W(z)+W(-z)}{2}\right]+\left[\frac{Y(z)+Y(-z)}{2}\right]\left[\frac{W(z)-W(-z)}{2}\right]\right\} \\
& =z[Y(z) W(z)-Y(-z) W(-z)],
\end{aligned}
$$

which is equivalent to (2.6). 
Note that since Theorem 1 holds for any $\phi \in C_{0}(\mathbb{R})$ satisfying (1.14), it also holds for any refinable function $\phi$ with refinement sequence $\left\{p_{j}\right\}$ satisfying (1.13).

In Section 3, we shall derive a sufficient condition on the Laurent polynomial $W$ for the corresponding approximation operator $\mathcal{L}_{r}$ to also possess the polynomial exactness property (1.10), as in (C) of Section 1, before establishing in Section 4 a constructive existence theory for the solution of a class of identities which includes (2.6) as a special case.

An analogous argument shows that if the interpolation point sequence $\left\{\frac{j}{2^{r-1}}\right.$ : $j \in \mathbb{Z}\}$ in (B) of Section 1 is replaced by the finer sequence $\left\{\frac{j}{2^{r}}: j \in \mathbb{Z}\right\}$, then the sequence $\left\{y_{j}\right\}$ in (1.11) must have precisely one non-zero value. An example is provided by the choice $\phi=h$, the shifted hat function, as defined by

$$
h(x):= \begin{cases}x, & x \in[0,1) ; \\ 2-x, & x \in[1,2) ; \\ 0, & x \in \mathbb{R} \backslash[0,2),\end{cases}
$$

which is a refinable function with refinement sequence

$$
\left\{p_{0}, p_{1}, p_{2}\right\}=\left\{\frac{1}{2}, 1, \frac{1}{2}\right\} ; \quad p_{j}=0, \quad j \in \mathbb{Z} \backslash\{0,1,2\},
$$

and in which case the corresponding local interpolation operator is the piecewise linear interpolant of any $f \in C(\mathbb{R})$ with respect to the interpolation point sequence $\left\{\frac{j}{2^{r}}: j \in \mathbb{Z}\right\}$.

Our examples in Sections 5, 6 and 7 are with respect to refinable functions $\phi$ for which the sequence $\left\{y_{j}\right\}$ in (1.11) has at least two non-zero values, in which case local interpolation at the sequence $\left\{\frac{j}{2^{r}}: j \in \mathbb{Z}\right\}$ is not possible, and where we shall rely on Theorem 1 for the construction of local interpolation operators with respect to the coarser sequence $\left\{\frac{j}{2^{r-1}}: j \in \mathbb{Z}\right\}$.

\section{Optimal POlynomial EXACTNESS}

For a refinable function $\phi$ with refinement sequence $\left\{p_{j}\right\}$ satisfying the support property (1.13) for an integer $\nu \geq 2$, as well as the sum-rule condition of order $m \geq 2$, let $\left\{w_{j}\right\} \in \ell_{0}(\mathbb{Z})$ denote a sequence such that, as in Theorem 1 , the compactly supported function $w$ in (2.1) satisfies the interpolatory condition (2.2). We proceed to establish a sufficient condition on $\left\{w_{j}\right\}$ for $w$ to satisfy, in addition, the property

$$
\sum_{j} f(j) w(2 x-2 j)=f(x), \quad x \in \mathbb{R}, \quad f \in \pi_{m-1},
$$

since then, for any $r \in \mathbb{Z}$ and $f \in \pi_{m-1}$, the polynomial $g(x):=f\left(\frac{x}{2^{r-1}}\right)$ satisfies $g \in \pi_{m-1}$, and it follows from (2.4) and (3.1) that, for $x \in \mathbb{R}$,

$$
\left(\mathcal{L}_{r} f\right)(x)=\sum_{j} g(j) w\left(2\left(2^{r-1} x\right)-2 j\right)=g\left(2^{r-1} x\right)=f(x) .
$$

Hence, if a sequence $\left\{w_{j}\right\} \in \ell_{0}(\mathbb{Z})$ is such that the compactly supported function $w$ in (2.1) satisfies (3.1), then the corresponding approximation operator $\mathcal{L}_{r}$ in (2.4) satisfies, for any $r \in \mathbb{Z}$, the optimal polynomial exactness condition (1.10) in (C) of Section 1.

In order to achieve (3.1), we now set

$$
w_{j}:=0, \quad j \notin\left\{-2\left\lfloor\frac{1}{2}(m+\nu-2)\right\rfloor, \ldots, 2\left\lfloor\frac{1}{2}(m+\nu-1)\right\rfloor-\nu\right\},
$$


with $\lfloor a\rfloor$ denoting the largest integer $\leq a$, so that from (2.1) we have

$$
w(x)=\sum_{j=-2\left\lfloor\frac{1}{2}(m+\nu-2)\right\rfloor}^{2\left\lfloor\frac{1}{2}(m+\nu-1)\right\rfloor-\nu} w_{j} \phi(x-j) .
$$

Since $\phi$ satisfies the support property (1.14), we deduce from (3.3) that

$$
\operatorname{supp}^{c} w \subset\left[-2\left\lfloor\frac{1}{2}(m+\nu-2)\right\rfloor, 2\left\lfloor\frac{1}{2}(m+\nu-1)\right\rfloor\right] .
$$

Moreover, for the Laurent polynomial $W$ defined in (2.5), it follows from (3.2) that

$$
W(z)=\frac{1}{2} \sum_{j} w_{j} z^{j}=\frac{1}{2} \sum_{j=-2\left\lfloor\frac{1}{2}(m+\nu-2)\right\rfloor}^{2\left\lfloor\frac{1}{2}(m+\nu-1)\right\rfloor-\nu} w_{j} z^{j} .
$$

Observe from (3.5) that the definition

$$
X(z):=z^{2\left\lfloor\frac{1}{2}(m+\nu-2)\right\rfloor} W(z)
$$

then implies that $X$ is a polynomial, with $X \in \pi_{2 m+\nu-4}$.

We proceed to establish in Theorem 2 below a sufficient condition on the polynomial $X$ for $w$ to satisfy (3.1). To this end, we first define, for any sequence $\mathbf{a}=\left\{a_{j}\right\} \in \ell_{0}(\mathbb{Z})$, its corresponding subdivision operator $\mathcal{S}_{\mathbf{a}}: \ell(\mathbb{Z}) \rightarrow \ell(\mathbb{Z})$ by

$$
\left(\mathcal{S}_{\mathbf{a}} \mathbf{c}\right)_{j}:=\sum_{k} a_{j-2 k} c_{k}, \quad j \in \mathbb{Z}, \quad \mathbf{c}=\left\{c_{j}\right\} \in \ell(\mathbb{Z}) .
$$

Also, for any non-negative integer $k$, we define the space of discrete polynomials of degree at most $k$ by

$$
\pi_{k}^{d}:=\left\{\left\{c_{j}\right\} \in \ell(\mathbb{Z}): c_{j}=f(j), \text { where } f \in \pi_{k}\right\} .
$$

Theorem 2. Let $\phi$ denote a refinable function with refinement sequence $\left\{p_{j}\right\}$ satisfying the sum-rule condition of order $m \geq 2$ and such that the support property (1.13) holds for an integer $\nu \geq 2$. Also, let $\left\{w_{j}\right\} \in \ell_{0}(\mathbb{Z})$ be a sequence such that, as in Theorem 1, the compactly supported function $w$ in (2.1) satisfies the interpolation property (2.2). Suppose, moreover, that the polynomial $X \in \pi_{2 m+\nu-4}$, as defined by (3.6), (3.5), satisfies the formulation

$$
X(z)=\left(\frac{1+z}{2}\right)^{m} \tilde{X}(z)
$$

for some polynomial $\tilde{X} \in \pi_{m+\nu-4}$. Then $w$ satisfies the property (3.1).

Proof. Let $f \in \pi_{m-1}$, and fix $x \in \mathbb{R}$. With the sequence $\mathbf{x}=\left\{x_{j}\right\} \in \ell_{0}(\mathbb{Z})$ defined by

$$
\frac{1}{2} \sum_{j} x_{j} z^{j}:=X(z),
$$

which, together with (3.6) and (3.5), yields

$$
w_{j}=x_{j+2\left\lfloor\frac{1}{2}(m+\nu-2)\right\rfloor}, \quad j \in \mathbb{Z},
$$

we may now apply (2.1) and (3.11), as well as the subdivision operator definition (3.7), to deduce that

$$
\sum_{j} f(j) w(2 x-2 j)=\sum_{k}\left(\mathcal{S}_{\mathbf{x}} \mathbf{c}\right)_{k} \phi(2 x-k)
$$


with the sequence $\mathbf{c}=\left\{c_{j}\right\} \in \ell(\mathbb{Z})$ defined by

$$
c_{j}:=f\left(j+\left\lfloor\frac{1}{2}(m+\nu-2)\right\rfloor\right), \quad j \in \mathbb{Z} .
$$

Since $f \in \pi_{m-1}$, the polynomial $\tilde{f}(x):=f\left(x+\left\lfloor\frac{1}{2}(m+\nu-2)\right\rfloor\right)$ also satisfies $\tilde{f} \in \pi_{m-1}$, and it follows from (3.8) and (3.13) that $\mathbf{c}=\left\{c_{j}\right\} \in \pi_{m-1}^{d}$.

Next, we use (1.19) and (1.16) to deduce that $Y(1)=1$. Also, (3.6) and (3.9) imply $W(-1)=X(-1)=0$, so that by setting $z=1$ in the identity (2.6) of Theorem 1, we obtain $W(1)=1$, and thus also, from (3.6), $X(1)=1$, which, together with (3.9), then yields $\tilde{X}(1)=1$.

Hence, according to (1.6) - (1.8), together with (3.9), (3.10), the sequence $\left\{x_{j}\right\} \in$ $\ell_{0}(\mathbb{Z})$ satisfies the sum-rule condition of order at least $m$. But then, from the result [2, Theorem 5.1.1], the subdivision operator $S_{\mathbf{x}}$ maps $\pi_{m-1}^{d}$ into itself, and thus

$$
\left(\mathcal{S}_{\mathbf{x}} \mathbf{c}\right)_{k}=g(k), \quad k \in \mathbb{Z},
$$

for some polynomial $g \in \pi_{m-1}$.

Next, since $f \in \pi_{m-1}$, and $\left\{p_{j}\right\}$ satisfies the sum-rule condition of order $m$, we may apply the commutator identity in [2, Theorem 5.3.1] to deduce from (3.12), (3.14) and (1.14) that

$$
\begin{aligned}
\sum_{j} f(j) w(2 x-2 j) & =\sum_{k} g(k) \phi(2 x-k)=\sum_{k} \phi(k) g(2 x-k) \\
& =\sum_{k=1}^{\nu-1} \phi(k) g(2 x-k)=: \tilde{g}(x) .
\end{aligned}
$$

Since $g \in \pi_{m-1}$, we see from (3.15) that also $\tilde{g} \in \pi_{m-1}$, and thus $f-\tilde{g} \in \pi_{m-1}$, for which it follows from (3.15) and (2.2) that $(f-\tilde{g})(k)=0, k \in \mathbb{Z}$. Hence, since the zero polynomial is the only polynomial in $\pi_{m-1}$ with infinitely many distinct zeros, we deduce that $\tilde{g}=f$, which, together with (3.15), proves the desired result (3.1).

According to the results of Sections 2 and 3, with particular reference to Theorems 1 and 2, we have now established the following result with respect to local interpolation with optimal polynomial reproduction in refinement spaces.

Corollary 1. For a refinable function $\phi$ with refinement sequence $\left\{p_{j}\right\}$ satisfying the sum-rule condition of order $m \geq 2$ and such that the support property (1.13) holds for an integer $\nu \geq 2$, let the polynomial $Y \in \pi_{\nu-2}$ be given by (1.19), (1.11). Also, let the sequence $\left\{w_{j}\right\} \in \ell_{0}(\mathbb{Z})$ be defined by

$$
\frac{1}{2} \sum_{j} w_{j} z^{j}:=z^{-2\left\lfloor\frac{1}{2}(m+\nu-2)\right\rfloor}\left(\frac{1+z}{2}\right)^{m} \tilde{X}(z),
$$

where $\tilde{X} \in \pi_{m+\nu-4}$ satisfies the polynomial identity

$$
\left(\frac{1+z}{2}\right)^{m} Y(z) \tilde{X}(z)-\left(\frac{1-z}{2}\right)^{m} Y(-z) \tilde{X}(-z)=z^{2\left\lfloor\frac{1}{2}(m+\nu-2)\right\rfloor-1}, \quad z \in \mathbb{C} .
$$

Then, for any $r \in \mathbb{Z}$, the approximation operator $\mathcal{L}_{r}: C(\mathbb{R}) \rightarrow S_{r}$, as formulated in (2.4), with the compactly supported function $w$ defined by (2.1), is a local interpolation operator with optimal polynomial exactness, as described in (A), (B) and (C) of Section 1. 
We proceed in Section 4 to establish a constructive existence theory for a class of polynomial identities which includes (3.17) as a special case.

\section{A general Class of Polynomial identities}

In this section, for given polynomials $G$ and $F$ satisfying certain conditions, we investigate the existence and construction of polynomial solutions $H$ of the identity

$$
G(z) H(z)-G(-z) H(-z)=z F\left(z^{2}\right), \quad z \in \mathbb{C} .
$$

For a polynomial $f$, if $f\left(z_{0}\right)=f\left(-z_{0}\right)=0$ for some $z_{0} \in \mathbb{C} \backslash\{0\}$, we call $z_{0}$ and $-z_{0}$ symmetric zeros of $f$. Also, for any function $f: \mathbb{C} \rightarrow \mathbb{C}$, we define $f_{-}(z):=f(-z)$.

Our following result for the polynomial identity (4.1), which extends the result of [2, Theorem 7.1.1], where a specific choice for $F$ was considered, is as follows.

Theorem 3. For polynomials $G$ and $F$ such that $d:=\operatorname{deg}(G) \geq 2, G(0) \neq 0$, $G$ has no symmetric zeros, and $F \in \pi_{d-1}$, where $F$ is not the zero polynomial, let $\{U, V\}$ and $\{Q, R\}$, with $R \in \pi_{d-1}$, denote the polynomial pairs obtained from, respectively, the Euclidean algorithm result

$$
G(z) U(z)+G(-z) V(z)=1, \quad z \in \mathbb{C},
$$

and the subsequent polynomial division result

$$
z V(z) F\left(z^{2}\right)=Q(z) G(z)+R(z), \quad z \in \mathbb{C} .
$$

Then:

(a) The polynomial

$$
H=\tilde{H}:=-R_{-} \in \pi_{d-1}
$$

satisfies the identity (4.11) and is the only solution in $\pi_{d-1}$ of (4.1).

(b) If $F \in \pi_{d-2}$, the polynomial $\tilde{H}$ in (a) satisfies $\tilde{H} \in \pi_{d-2}$.

(c) If $H=H^{*}$ is any polynomial solution of the identity (4.1), the general solution of (4.1) is given by

$$
H(z)=H^{*}(z)+J\left(z^{2}\right) G(-z),
$$

with $J$ denoting an arbitrary polynomial.

Proof. (a) With the definition $\tilde{F}(z):=z F\left(z^{2}\right)$, we first multiply the identity (4.2) by $\tilde{F}$ and apply (4.3) to deduce that

$$
G \tilde{H}+G_{-} R=\tilde{F},
$$

where $\tilde{H}:=U \tilde{F}+Q G_{-}$. If both $\tilde{H}$ and $R$ are not the zero polynomial, it follows from (4.6) that

$$
\begin{aligned}
d+\operatorname{deg}(\tilde{H}) & =\operatorname{deg}(G \tilde{H}) \leq \max \left\{\operatorname{deg}(\tilde{F}), \operatorname{deg}\left(G_{-} R\right)\right\} \\
& \leq \max \{2 d-1, d+\operatorname{deg}(R)\}=2 d-1,
\end{aligned}
$$

since $R \in \pi_{d-1}$, and thus $\operatorname{deg}(\tilde{H}) \leq d-1$, whereas if $\tilde{H}$ is not the zero polynomial and $R$ is the zero polynomial, then (4.6) yields $G \tilde{H}=\tilde{F}$, and it follows similarly that $\operatorname{deg}(\tilde{H}) \leq d-1$. We have therefore shown that $\tilde{H} \in \pi_{d-1}$.

Next, note from (4.6) that $G R_{-}+G_{-} \tilde{H}_{-}=\tilde{F}_{-}=-\tilde{F}$, which, when added to (4.6), yields

$$
G\left(\tilde{H}+R_{-}\right)=-G_{-}\left(\tilde{H}_{-}+R\right)
$$


Since, moreover, $\operatorname{gcd}\left\{G, G_{-}\right\}=1$, we deduce from (4.7) that

$$
\tilde{H}+R_{-}=K G_{-},
$$

for some polynomial $K$.

But $\tilde{H} \in \pi_{d-1}$ and $R \in \pi_{d-1}$ imply that $\tilde{H}+R_{-} \in \pi_{d-1}$, which, together with (4.8) and $\operatorname{deg}\left(G_{-}\right)=d$, show that $K$ is the zero polynomial, so that (4.8) yields $\tilde{H}=-R_{-}$or, equivalently, $R=-\tilde{H}_{-}$, which we can now substitute into (4.6) to deduce that the polynomial $H=\tilde{H}$ in (4.4) does indeed satisfy (4.1).

Next, suppose $H=\hat{H} \in \pi_{d-1}$ satisfies (4.1), according to which, together with the fact that $H=\tilde{H}$ also satisfies (4.1), we have $G(\tilde{H}-\hat{H})=G_{-}\left(\tilde{H}_{-}-\hat{H}_{-}\right)$, and thus, since $\operatorname{gcd}\left\{G, G_{-}\right\}=1$, it holds that $\tilde{H}-\hat{H}=L G_{-}$, for some polynomial $L$. Since $\tilde{H}-\hat{H} \in \pi_{d-1}$ and $\operatorname{deg}\left(G_{-}\right)=d$, it follows that $L$ must be the zero polynomial, and thus $\tilde{H}=\hat{H}$, which proves the uniqueness statement.

(b) Suppose $F \in \pi_{d-2}$, and let the coefficient sequences $\left\{g_{j}: j=0, \ldots, d\right\}$ and $\left\{\tilde{h}_{j}: j=0, \ldots, d-1\right\}$ be defined by

$$
\sum_{j=0}^{d} g_{j} z^{j}:=G(z) ; \quad \sum_{j=0}^{d-1} \tilde{h}_{j} z^{j}:=\tilde{H}(z),
$$

and where $\operatorname{deg}(G)=d$ gives $g_{d} \neq 0$. According to (a), we may substitute (4.9) into (4.1) to obtain

$$
2 g_{d} \tilde{h}_{d-1} z^{2 d-1}+Q(z)=\tilde{F}(z), \quad z \in \mathbb{C},
$$

with $Q \in \pi_{2 d-3}$, and where, since $F \in \pi_{d-2}$, we have $\tilde{F} \in \pi_{2 d-3}$. Since also $g_{d} \neq 0$, it then follows from (4.10) that $\tilde{h}_{d-1}=0$, and thus, from (4.9), $\tilde{H} \in \pi_{d-2}$.

(c) Let $H=H^{*}$ be a particular polynomial solution of (4.1). It follows that if $H$ is any polynomial solution (4.1), then

$$
G\left(H-H^{*}\right)=G_{-}\left(H_{-}-H_{-}^{*}\right),
$$

and thus, since $\operatorname{gcd}\left\{G, G_{-}\right\}=1$, we have

$$
H-H^{*}=\tilde{J} G_{-},
$$

for some polynomial $\tilde{J}$. By substituting (4.12) into (4.11), we obtain $G \tilde{J} G_{-}=$ $G_{-} \tilde{J}_{-} G$, which yields $\tilde{J}=\tilde{J}_{-}$, that is, $\tilde{J}$ is an even polynomial, and thus, from (4.12), $H$ is given by (4.5), for some polynomial $J$. Finally, observe that, for any polynomial $J$, the polynomial $H$ given by (4.5) satisfies the identity (4.1), which completes our proof.

In [2, Theorem 7.1.1], the special case

$$
F(z):=z^{\lfloor d / 2\rfloor-1}
$$

of Theorem 3 was established. Observe that (4.13) implies $F \in \pi_{d-2}$, so that Theorem 3(b) applies, that is, $\tilde{H} \in \pi_{d-2}$, as was also established in [2, Theorem 7.1.1]. For the further specialized case where, in addition to the choice (4.13) for $F$ the polynomial $G$ in Theorem 3 is chosen, for any $n \in \mathbb{N}$, as

$$
G(z):=\left(\frac{1+z}{2}\right)^{2 n},
$$


so that $d=2 n$, and thus, from (4.13),$F(z)=z^{n-1}$, an explicit formulation for the corresponding polynomial $\tilde{H} \in \pi_{2 n-2}$ of Theorem 3 was shown in [6] (see also [7], [2, Theorem 7.2.3]) to be given by

$$
\tilde{H}(z)=\tilde{H}_{2 n}(z):=z^{n-1} \sum_{j=0}^{n-1}\left(\begin{array}{c}
n+j-1 \\
j
\end{array}\right)\left[\frac{1}{2}\left(1-\frac{z+z^{-1}}{2}\right)\right]^{j},
$$

so that, moreover, $\operatorname{deg}\left(\tilde{H}_{2 n}\right)=2 n-2$.

In Sections 5 and 6 below, we shall apply Theorem 3 in two different contexts.

\section{Explicit CONSTRUCTION OF THE APPROXIMATION OPERATOR}

The result of Theorem 4 enables us to prove the following.

Theorem 4. In Corollary 1, suppose that, moreover, $\operatorname{deg}(Y)=\nu-2, Y(0) \neq 0$, and the polynomal $Y$ has no symmetric zeros. Then there exists a polynomial $\tilde{H} \in \pi_{m+\nu-4}$ satisfying the identity (3.17), and $\tilde{H}$ is the only solution in $\pi_{m+\nu-3}$ of (3.17).

Proof. Let the polynomials $G$ and $F$ be defined by

$$
G(z):=\left(\frac{1+z}{2}\right)^{m} Y(z) ; \quad F(z):=z^{\left\lfloor\frac{1}{2}(m+\nu-2)\right\rfloor-1} .
$$

Since $Y(1)=1$ and $Y$ has no symmetric zeros, it follows from (5.1) that $G$ has no symmetric zeros. Also, (5.1), together with $\operatorname{deg}(Y)=\nu-2$, shows that here $d:=\operatorname{deg}(G)=m+\nu-2$, whereas $Y(0) \neq 0$ yields $G(0) \neq 0$, with, moreover, $\operatorname{deg}(F) \leq \frac{1}{2}(m+\nu-2)-1=\frac{1}{2} d-1 \leq d-2$, since $d \geq 2$, and thus $F \in \pi_{d-2}$.

Hence we may apply Theorem 3(a) and (b) to deduce that there exists a polynomial $H=\tilde{H} \in \pi_{m+\nu-4}$ satisfying the identity (4.1), which is the only solution in $\pi_{m+\nu-3}$ of (4.1). But, according to (5.1), the identities (4.1) and (3.17) are equivalent, so that our proof is completed by defining $\tilde{X}:=\tilde{H}$.

The results of Theorems 3 and 4 yield the following algorithm for the explicit construction of the approximation operator $\mathcal{L}_{r}$ of Corollary 1.

Algorithm 1 (Explicit construction of $\mathcal{L}_{r}$ ). Let $\phi$ be a refinable function with refinement sequence $\left\{p_{j}\right\}$ satisfying the sum-rule condition of order $m \geq 2$, as well as $\operatorname{supp}\left\{p_{j}\right\}=\left.[0, \nu]\right|_{\mathbb{Z}}$ for an integer $\nu \geq 2$, and where the polynomial $Y(z):=$ $\sum_{j=0}^{\nu-2} \phi(j+1) z^{j}$ satisfies $\operatorname{deg}(Y)=\nu-2, Y(0) \neq 0$, and $Y$ has no symmetric zeros.

1. Apply the Euclidean algorithm to obtain the polynomial pair $\{U, V\}$ such that

$$
\left(\frac{1+z}{2}\right)^{m} Y(z) U(z)+\left(\frac{1-z}{2}\right)^{m} Y(-z) V(z)=1, \quad z \in \mathbb{C} .
$$

2. Apply polynomial division to obtain the polynomial pair $\{Q, R\}$, with $R \in$ $\pi_{m+\nu-4}$, such that

$$
z^{2\left\lfloor\frac{1}{2}(m+\nu-2)\right\rfloor-1} V(z)=Q(z)\left(\frac{1+z}{2}\right)^{m} Y(z)+R(z), \quad z \in \mathbb{C} .
$$


3. Define the sequence $\left\{w_{j}\right\} \in \ell_{0}(\mathbb{Z})$ by

$$
\left.\begin{array}{rl}
\sum_{j=-2\left\lfloor\frac{1}{2}(m+\nu-2)\right\rfloor}^{2\left\lfloor\frac{1}{2}(m+\nu-1)\right\rfloor-\nu} w_{j} z^{j} & :=-2 z^{-2\left\lfloor\frac{1}{2}(m+\nu-2)\right\rfloor}\left(\frac{1+z}{2}\right)^{m} R(-z) ; \\
w_{j} & :=0, \quad j \notin\left\{-2\left\lfloor\frac{1}{2}(m+\nu-2)\right\rfloor, \ldots, 2\left\lfloor\frac{1}{2}(m+\nu-1)\right\rfloor-\nu\right\} .
\end{array}\right\}
$$

4. Define, for any $f \in C(\mathbb{R})$ and $r \in \mathbb{Z}$,

$$
\left(\mathcal{L}_{r} f\right)(x):=\sum_{j}\left[\sum_{k} w_{j-2 k} f\left(\frac{k}{2^{r-1}}\right)\right] \phi\left(2^{r} x-j\right) .
$$

We proceed, in Example A below, to consider the case where $\phi=N_{m}$, the cardinal $B$-spline of order $m \geq 2$.

Example A (Cardinal $B$-splines). For any integer $m \geq 2$, let $\phi=N_{m}$, where the cardinal $B$-splines $\left\{N_{m}: m=2,3, \ldots\right\}$ are defined recursively by

$$
N_{2}(x):=h(x) ; \quad N_{m+1}(x):=\int_{0}^{1} N_{m}(x-t) d t, \quad m=2,3, \ldots,
$$

with $h$ denoting the shifted hat function, as given by (2.8). Then, as proved in [3. Chapters 4 and 6] (see also [12], 2, Chapter 2]), $\phi$ is a refinable function with refinement sequence $\left\{p_{j}\right\}=\left\{p_{m, j}\right\}$ given by

$$
p_{m, j}:=\frac{1}{2^{m-1}}\left(\begin{array}{c}
m \\
j
\end{array}\right), \quad j \in \mathbb{Z},
$$

where $\left(\begin{array}{c}m \\ j\end{array}\right):=0, j \notin\{0, \ldots, m\} ; \phi$ satisfies the support property (1.14), with $\nu=m$, as well as the condition (1.12); $\phi$ is strictly positive in $(0, m) ; \phi$ possesses $m-2$ continuous derivatives on $\mathbb{R}$; for any $r \in \mathbb{Z}$, the linear space $S_{r}=S_{r}^{m}$ in (1.2) satisfies

$$
S_{r}^{m}=\left\{s \in C^{m-2}(\mathbb{R}):\left.s\right|_{\left[\frac{j}{2^{r}}, \frac{j+1}{2^{r}}\right)} \in \pi_{m-1}, j \in \mathbb{Z}\right\}, \quad r \in \mathbb{Z}
$$

the polynomial $Y=Y_{m}$, as in (1.19), (1.11), in this case known as the EulerFrobenius polynomial, has only negative zeros and therefore possesses no symmetric zeros, with also $\operatorname{deg}\left(Y_{m}\right)=m-2$ and $Y_{m}(0) \neq 0$.

Observe also from (5.7) that

$$
P_{m}(z):=\frac{1}{2} \sum_{j} p_{m, j} z^{j}=\frac{1}{2} \sum_{j=0}^{m} p_{m, j} z^{j}=\left(\frac{1+z}{2}\right)^{m},
$$

according to which the cardinal $B$-spline refinement sequence $\left\{p_{j}\right\}=\left\{p_{m, j}\right\}$ satisfies the sum-rule condition of order $m$.

Hence we may apply Theorem 4 to deduce the existence of a solution $\tilde{X} \in \pi_{2 m-4}$ of the identity (3.17) in Corollary 1, as given here by

$$
\left(\frac{1+z}{2}\right)^{m} Y_{m}(z) \tilde{X}(z)-\left(\frac{1-z}{2}\right)^{m} Y_{m}(-z) \tilde{X}(-z)=z^{2 m-3}, \quad z \in \mathbb{C},
$$


and where $\tilde{X}$ is the only solution in $\pi_{2 m-3}$ of (5.10). The corresponding cardinal spline approximation operator $\mathcal{L}_{r}=\mathcal{L}_{m, r}: C(\mathbb{R}) \rightarrow S_{r}^{m}$, as obtained from Algorithm 1, is then a local interpolation operator, as in (A) and (B) of Section 1, with polynomial exactness on $\pi_{m-1}$.

By recalling also from [3, Chapter 4] the explicit formulation

$$
N_{m}(j)=\frac{1}{(m-1) !} \sum_{k=0}^{j-1}(-1)^{k}\left(\begin{array}{c}
m \\
k
\end{array}\right)(j-k)^{m-1}, \quad j=1, \ldots, m-1,
$$

we now proceed to explicitly calculate the cubic $(m=4)$ and quadratic $(m=3)$ cardinal spline cases.

The cubic case $m=4$. First, we use (5.11), (1.19) and (1.11) to obtain the EulerFrobenius polynomial $Y_{4}(z)=\frac{1}{6}\left(1+4 z+z^{2}\right)$. Next, following Step 1 of Algorithm 1 , we apply the Euclidean algorithm to obtain polynomials $U$ and $V$ satisfying the identity (5.2), that is,

$$
\left(\frac{1+z}{2}\right)^{4}\left[\frac{1}{6}\left(1+4 z+z^{2}\right)\right] U(z)+\left(\frac{1-z}{2}\right)^{4}\left[\frac{1}{6}\left(1-4 z+z^{2}\right)\right] V(z)=1, \quad z \in \mathbb{C},
$$

and thereby yielding

$$
\left.\begin{array}{l}
U(z)=\frac{1}{6}\left(288-1013 z+1480 z^{2}-1078 z^{3}+376 z^{4}-47 z^{5}\right) \\
V(z)=\frac{1}{6}\left(288+1013 z+1480 z^{2}+1078 z^{3}+376 z^{4}+47 z^{5}\right)
\end{array}\right\}
$$

As in Step 2 of Algorithm 1, we next apply polynomial division to obtain the polynomials $Q$ and $R$, with $R \in \pi_{4}$, such that, as in (5.3) with $m=\nu=4$,

$$
z^{5} V(z)=Q(z)\left(\frac{1+z}{2}\right)^{4}\left[\frac{1}{6}\left(1+4 z+z^{2}\right)\right]+R(z), \quad z \in \mathbb{C},
$$

which gives

$$
\left.\begin{array}{l}
Q(z)=16\left(1-3 z^{2}+47 z^{4}\right) \\
R(z)=-\frac{1}{6}\left(1+8 z+20 z^{2}+8 z^{3}+z^{4}\right)
\end{array}\right\}
$$

By applying the definition (5.4) in Step 3 of Algorithm 1, we obtain

$$
\left.\begin{array}{c}
\left\{w_{-6}, \ldots, w_{2}\right\}=\left\{\frac{1}{48},-\frac{4}{48},-\frac{6}{48}, \frac{28}{48}, \frac{58}{48}, \frac{28}{48},-\frac{6}{48},-\frac{4}{48}, \frac{1}{48}\right\} ; \\
w_{j}=0, \quad j \notin\{-6, \ldots, 2\},
\end{array}\right\}
$$

which, together with (5.5), then yields an explicit formulation of the local interpolation operator $\mathcal{L}_{r}=\mathcal{L}_{4, r}: C(\mathbb{R}) \rightarrow S_{r}^{4}$, with exactness on the polynomial space $\pi_{3}$.

The quadratic case $m=3$. From (5.11), (1.19) and (1.11), we obtain the EulerFrobenius polynomial $Y(z)=E_{3}(z)=\frac{1}{2}(1+z)$, according to which, together with $m=\nu=3$, the polynomial identity (3.17) in Corollary 1 is given here by

$$
\left(\frac{1+z}{2}\right)^{4} \tilde{X}(z)-\left(\frac{1-z}{2}\right)^{4} \tilde{X}(-z)=z^{3}, \quad z \in \mathbb{C} .
$$

Hence we may proceed here more efficiently than in Algorithm 1 by deducing directly from the formula (4.15), with $n=2$, that the solution $\tilde{X} \in \pi_{2}$ of the polynomial identity (5.12) is given by

$$
\tilde{X}(z)=-\frac{1}{2}\left(1-4 z+z^{2}\right)
$$


and then using (3.16) and (5.13) to obtain

$$
\left\{w_{-4}, \ldots, w_{1}\right\}=\left\{-\frac{1}{8}, \frac{1}{8}, 1,1, \frac{1}{8},-\frac{1}{8}\right\} ; \quad w_{j}=0, \quad j \notin\{-4, \ldots, 1\},
$$

which, together with (5.5), then yields an explicit formulation of the local interpolation operator $\mathcal{L}_{r}=\mathcal{L}_{3, r}: C(\mathbb{R}) \rightarrow S_{r}^{3}$, with exactness on the polynomial space $\pi_{2}$.

Remark. Note that Algorithm 1 requires as input the values $\left\{y_{j}=\phi(j): j=\right.$ $1, \ldots, \nu-1\}$, which are then used in the applications of the Euclidean algorithm and polynomial division in, respectively, Steps 1 and 2. For the cardinal $B$-spline examples in Example A above, as based on the explicit formulation (5.11), as well as on Example B in Section 6 below and also the example in the final paragraph of Section 7 below, the $y_{j}$-values are either calculable or prescribed as rational numbers, so that Steps 1 and 2 may be performed in exact rational arithmetic, yielding the desired weights $w_{j}$ in Step 3 in precise rational form. For those cases where the $y_{j}$-values need to be computed numerically from the eigenvalue problem (1.18) and are therefore subject to small errors, Algorithm 1 needs further investigation with respect to its numerical stability in Steps 1 and 2.

\section{Prescribed Refinable function values on $\mathbb{Z}$}

In the polynomial identity (3.17) of Corollary 1 , the polynomial $Y$ is obtained by means of (1.19) and (1.11) from a given refinable function $\phi$. In this section, as further development of the results in [8, we consider the inverse problem of establishing sufficient conditions on a sequence $\left\{y_{j}\right\} \in \ell_{0}(\mathbb{Z})$ for the existence of a refinable function $\phi$ such that

$$
\phi(j)=y_{j}, \quad j \in \mathbb{Z}
$$

As a first step in this direction, we prove the following necessary condition on $\left\{y_{j}\right\}$.

Theorem 5. Let $\left\{y_{j}\right\} \in \ell_{0}(\mathbb{Z})$ be a sequence for which there exists a refinable function $\phi$ satisfying the condition (6.1). Then the refinement sequence $\left\{p_{j}\right\}$ of $\phi$ satisfies the condition

$$
\sum_{k} y_{2 j-k} p_{k}=y_{j}, \quad j \in \mathbb{Z}
$$

which, with the definitions

$$
Y(z):=\sum_{j} y_{j+1} z^{j} ; \quad P(z):=\frac{1}{2} \sum_{j} p_{j} z^{j}
$$

can equivalently be formulated as the identity

$$
Y(z) P(z)-Y(-z) P(-z)=z Y\left(z^{2}\right), \quad z \in \mathbb{C} \backslash\{0\} .
$$

Proof. As noted before in (1.17), the condition (6.2) is an immediate consequence of (1.1) and (6.1). By applying the definitions in (6.3), we next deduce that the 
condition (6.2) is satisfied if and only if, for any $z \in \mathbb{C} \backslash\{0\}$,

$$
\begin{aligned}
z Y\left(z^{2}\right) & =\sum_{j}\left[\sum_{k} y_{2 j-2 k} p_{2 k}+\sum_{k} y_{2 j-2 k-1} p_{2 k+1}\right] z^{2 j-1} \\
& =\left[\sum_{j} y_{2 j} z^{2 j-1}\right]\left[\sum_{k} p_{2 k} z^{2 k}\right]+\left[\sum_{j} y_{2 j+1} z^{2 j}\right]\left[\sum_{k} p_{2 k+1} z^{2 k+1}\right] \\
& =\frac{1}{2}[Y(z)-Y(-z)][P(z)+P(-z)]+\frac{1}{2}[Y(z)+Y(-z)][P(z)-P(-z)] \\
& =Y(z) P(z)-Y(-z) P(-z),
\end{aligned}
$$

which yields (6.4), thereby completing our proof.

For a given sequence $\left\{y_{j}\right\} \in \ell_{0}(\mathbb{Z})$ satisfying certain conditions, we now apply Theorem 3 to obtain, as stated in the following theorem, a sequence $\left\{p_{j}\right\} \in \ell_{0}(\mathbb{Z})$ satisfying the condition (6.2) in Theorem 5, as well as the sum-rule condition of order $\geq 2$.

Theorem 6. Suppose $\left\{y_{j}\right\} \in \ell_{0}(\mathbb{Z})$ is a sequence satisfying the conditions (1.15) and (1.16) for an integer $\nu \geq 2$, and such that the polynomial $Y \in \pi_{\nu-2}$, as defined in (6.3), satisfies $\operatorname{deg}(Y)=\nu-2, Y(0) \neq 0$, and $Y$ has no symmetric zeros. Then there exists precisely one sequence $\left\{p_{j}\right\} \in \ell_{0}(\mathbb{Z})$, with

$$
\left.\operatorname{supp}\left\{p_{j}\right\} \subset[0, \nu]\right|_{\mathbb{Z}}
$$

such that $\left\{p_{j}\right\}$ satisfies the condition (6.2) of Theorem 5, as well as the sum-rule condition of order at least 2. Moreover, $\left\{p_{j}\right\}$ satisfies the formulation

$$
\frac{1}{2} \sum_{j} p_{j} z^{j}=\frac{1}{2} \sum_{j=0}^{\nu} p_{j} z^{j}=\left(\frac{1+z}{2}\right)^{2} \tilde{H}(z),
$$

with the polynomial $\tilde{H} \in \pi_{\nu-2}$ obtained as in (4.4) of Theorem 3 , with $d=\nu$, and

$$
G(z):=\left(\frac{1+z}{2}\right)^{2} Y(z) ; \quad F(z):=Y(z)
$$

Proof. First, note from (1.16) and (6.3) that $Y(1)=1$. Since, moreover, $\operatorname{deg}(Y)=$ $\nu-2 ; Y(0) \neq 0$, and $Y$ has no symmetric zeros, it follows that the polynomials $G$ and $F$ in (6.7) satisfy the conditions of Theorem 3, with $d=\nu$ and $F \in \pi_{\nu-2}$. With $\tilde{H}$ denoting the polynomial defined in (4.4), it then follows from Theorem 3(b) that $\tilde{H} \in \pi_{\nu-2}$. Moreover, it follows from Theorem 3(a), together with (6.7), that

$$
Y(z)\left[\left(\frac{1+z}{2}\right)^{2} \tilde{H}(z)\right]-Y(-z)\left[\left(\frac{1-z}{2}\right)^{2} \tilde{H}(-z)\right]=z Y\left(z^{2}\right), \quad z \in \mathbb{C},
$$

according to which the polynomial

$$
P(z):=\left(\frac{1+z}{2}\right)^{2} \tilde{H}(z)
$$

satisfies the identity (6.4) of Theorem 5, and thus, by applying Theorem 5, we deduce that the sequence $\left\{p_{j}\right\} \in \ell_{0}(\mathbb{Z})$, as defined by (6.6), satisfies the condition (6.2). 
Since $Y(1)=1$, we may set $z=1$ in 6.8 to obtain $\tilde{H}(1)=1$, and it follows from the definition of the polynomial $P$ in 6.3 that the sequence $\left\{p_{j}\right\}$ satisfies the sum-rule condition of order at least 2. Finally, observe that the uniqueness statement with respect to $\left\{p_{j}\right\}$ is a consequence of the uniqueness statement in Theorem 3(a).

The results of Theorems 6 and 3 yield the following algorithm for the explicit construction of the sequence $\left\{p_{j}\right\}$ of Theorem 6 .

Algorithm 2 (Explicit construction of $\left\{p_{j}\right\}$ from $\left.\left\{y_{j}\right\}\right)$. Let $\left\{y_{j}\right\} \in \ell_{0}(\mathbb{Z})$ be a sequence such that $\operatorname{supp}\left\{y_{j}\right\}=\left.[1, \nu-1]\right|_{\mathbb{Z}}$ for an integer $\nu \geq 2$, with also $\sum_{j=1}^{\nu-1} y_{j}=1$, and where the polynomial

$$
Y(z):=\sum_{j=0}^{\nu-2} y_{j+1} z^{j} \text { has no symmetric zeros. }
$$

1. Apply the Euclidean algorithm to obtain the polynomial pair $\{U, V\}$ such that

$$
\left[\left(\frac{1+z}{2}\right)^{2} Y(z)\right] U(z)+\left[\left(\frac{1-z}{2}\right)^{2} Y(-z)\right] V(z)=1, \quad z \in \mathbb{C} .
$$

2. Apply polynomial division to obtain the polynomial pair $\{Q, R\}$, with $R \in$ $\pi_{\nu-2}$, such that

$$
z Y\left(z^{2}\right) V(z)=Q(z)\left(\frac{1+z}{2}\right)^{2} Y(z)+R(z), \quad z \in \mathbb{C} .
$$

3. The sequence $\left\{p_{j}\right\} \in \ell_{0}(\mathbb{Z})$ of Theorem 6 is then given by

$$
\sum_{j=0}^{\nu} p_{j} z^{j}:=-2\left(\frac{1+z}{2}\right)^{2} R(-z) .
$$

We proceed to establish, in Theorem 7 below, a set of sufficient conditions on a sequence $\left\{y_{j}\right\} \in \ell_{0}(\mathbb{Z})$ for the existence of a refinable function $\phi$ with refinement sequence $\left\{p_{j}\right\}$ as given in Theorem 6 , and where $\phi$ satisfies the condition (6.1). We shall rely on the following definition of subdivision convergence, as was given in [2, Chapter 4].

For a sequence $\mathbf{p}=\left\{p_{j}\right\} \in \ell_{0}(\mathbb{Z})$, the subdivision operator $\mathcal{S}_{\mathbf{p}}: \ell(\mathbb{Z}) \rightarrow \ell(\mathbb{Z})$, as defined in (3.7), is said to provide a convergent subdivision scheme if there exists a non-trivial function $\phi \in C(\mathbb{R})$, called the limit function corresponding to $\mathcal{S}_{\mathbf{p}}$, such that

$$
\sup _{j}\left|\phi\left(\frac{j}{2^{r}}\right)-\left(\mathcal{S}_{\mathbf{p}}^{r} \boldsymbol{\delta}\right)_{j}\right| \rightarrow 0, \quad r \rightarrow \infty,
$$

where $\boldsymbol{\delta}:=\left\{\delta_{j}\right\}$, as in (2.3).

Theorem 7. Let $\left\{y_{j}\right\} \in \ell_{0}(\mathbb{Z})$ denote a sequence satisfying the conditions of Theorem 6 and such that, moreover, for the sequence $\mathbf{p}=\left\{p_{j}\right\} \in \ell_{0}(\mathbb{Z})$ of Theorem 6 , the subdivision operator $\mathcal{S}_{\mathbf{p}}$ provides a convergent subdivision scheme. Then the limit function $\phi$ corresponding to $\mathcal{S}_{\mathbf{p}}$ is a refinable function with refinement sequence $\left\{p_{j}\right\}$, and $\phi$ satisfies the condition (6.1). 
Proof. Since $\mathcal{S}_{\mathbf{p}}$ provides a convergent subdivision scheme, we know from a standard result (see [1] or [2, Chapter 4]) that $\phi$ is a refinable function with refinement sequence $\left\{p_{j}\right\}$ and such that (1.13) is satisfied.

Let $j \in \mathbb{Z}$ be fixed. In order to prove (6.1), we shall first show that

$$
y_{j}=\sum_{k}\left(\mathcal{S}_{\mathbf{p}}^{r} \boldsymbol{\delta}\right)_{2^{r} j-k} y_{k}, \quad r \in \mathbb{N},
$$

where $\boldsymbol{\delta}:=\left\{\delta_{j}\right\}$, as in (2.3). To this end, for any fixed $r \in \mathbb{N}$, we apply (3.7) and (6.2) to obtain

$$
\begin{aligned}
\sum_{k}\left(\mathcal{S}_{\mathbf{p}}^{r+1} \boldsymbol{\delta}\right)_{2^{r+1} j-k} y_{k} & =\sum_{k}\left[\sum_{\ell} p_{k-2 \ell}\left(\mathcal{S}_{\mathbf{p}}^{r} \boldsymbol{\delta}\right)_{\ell}\right] y_{2^{r+1} j-k} \\
& =\sum_{\ell}\left(\mathcal{S}_{\mathbf{p}}^{r} \boldsymbol{\delta}\right)_{\ell}\left[\sum_{k} y_{2\left(2^{r} j-\ell\right)-k} p_{k}\right] \\
& =\sum_{\ell}\left(\mathcal{S}_{\mathbf{p}}^{r} \boldsymbol{\delta}\right)_{\ell} y_{2^{r} j-\ell}=\sum_{k}\left(\mathcal{S}_{\mathbf{p}}^{r} \boldsymbol{\delta}\right)_{2^{r} j-k} y_{k}
\end{aligned}
$$

By observing from (6.2) that

$$
y_{j}=\sum_{k} y_{2 j-k}\left(\mathcal{S}_{\mathbf{p}} \boldsymbol{\delta}\right)_{k}=\sum_{k}\left(\mathcal{S}_{\mathbf{p}} \boldsymbol{\delta}\right)_{2 j-k} y_{k},
$$

we deduce that (6.14) holds for $r=1$. The desired result (6.14) then follows inductively from (6.15).

By applying (6.15), (1.15) and (1.16), we deduce that, for any $r \in \mathbb{Z}$,

$$
\begin{aligned}
& \left|y_{j}-\phi(j)\right|=\left|\sum_{k=1}^{\nu-1}\left[\left(\mathcal{S}_{\mathbf{p}}^{r} \boldsymbol{\delta}\right)_{2^{r} j-k}-\phi(j)\right] y_{k}\right| \\
& \leq \max _{1 \leq k \leq \nu-1}\left|y_{k}\right|\left\{\sum_{k=1}^{\nu-1}\left|\phi\left(\frac{2^{r} j-k}{2^{r}}\right)-\left(\mathcal{S}_{\mathbf{p}}^{r} \boldsymbol{\delta}\right)_{2^{r} j-k}\right|+\sum_{k=1}^{\nu-1}\left|\phi(j)-\phi\left(j-\frac{k}{2^{r}}\right)\right|\right\} \\
& \leq(\nu-1) \max _{1 \leq k \leq \nu-1}\left|y_{k}\right|\left\{\sup _{k}\left|\phi\left(\frac{k}{2^{r}}\right)-\left(\mathcal{S}_{\mathbf{p}}^{r} \boldsymbol{\delta}\right)_{k}\right|+\max _{1 \leq k \leq \nu-1}\left|\phi(j)-\phi\left(j-\frac{k}{2^{r}}\right)\right|\right\} \\
& \rightarrow 0, r \rightarrow \infty,
\end{aligned}
$$

from (6.13), as well as the fact that $\phi$ is continuous at $j$, thereby showing that the condition (6.1) is satisfied.

In Example B below, we shall use the fact, as proved in [10, Theorem 2.5] (see also [2, Corollary 6.4.1]), that if $\left\{p_{j}\right\} \in \ell_{0}(\mathbb{Z})$ is a sequence satisfying the support property (1.13) for an integer $\nu \geq 2$, as well as the sum rule and the positivity condition

$$
p_{j}>0, \quad j=0, \ldots, \nu,
$$

then the subdivision operator $\mathcal{S}_{\mathbf{p}}$ provides a convergent subdivision scheme. 
In addition, we shall rely on the fact, as established in [2, Corollary 4.5.2], that if $\phi$ is a refinable function with refinement sequence $\left\{p_{j}\right\}$ satisfying the sum rule and where $\phi$ satisfies the condition (1.12), then $\phi$ is the only function in $C_{0}(\mathbb{R})$ satisfying both (1.1) and (1.12).

For any non-negative integer $k$ and $\alpha \in(0,1]$, we shall denote by $C_{0}^{k, \alpha}(\mathbb{R})$ the Hölder continuity space defined as the set of functions $f \in C_{0}^{k}(\mathbb{R})$ satisfying the condition

$$
\left|f^{(k)}(x)-f^{(k)}(y)\right| \leq c|x-y|^{\alpha}, \quad x, y \in \mathbb{R},
$$

for some constant $c$. As proved in [2, Theorem 6.4.3], if a sequence $\left\{p_{j}\right\} \in \ell_{0}(\mathbb{Z})$ satisfies the support property (1.13) for an integer $\nu \geq 2$ and the positivity condition (6.16), as well as the sum-rule condition of order $m \in\{1, \ldots, \nu-1\}$, then, with $n$ denoting the smallest integer in the set $\{1, \ldots, m\}$ such that the sequence $\left\{\tilde{p}_{j}\right\} \in$ $\ell_{0}(\mathbb{Z})$ defined by

$$
\sum_{j} \tilde{p}_{j} z^{j}:=\left(\frac{1+z}{2}\right)^{n-m} \sum_{j} p_{j} z^{j}
$$

satisfies the condition

$$
\tilde{p}_{j}>0, \quad j=0, \ldots, \nu-m+n,
$$

the limit (refinable) function $\phi$ corresponding to the (convergent) subdivision operator $\mathcal{S}_{\mathbf{p}}$ satisfies the Hölder regularity result

$$
\phi \in C_{0}^{m-n, \alpha}(\mathbb{R}), \quad \text { where } \quad \alpha:=\log _{2}\left[\frac{1}{1-\min \left\{\tilde{p}_{0}, \ldots, \tilde{p}_{\nu-m+n}\right\}}\right] .
$$

Example B. Let $\left\{y_{j}\right\} \in \ell_{0}(\mathbb{Z})$ be defined by

$$
\left\{y_{1}, y_{2}, y_{3}\right\}=\left\{\frac{1}{5}, \frac{3}{5}, \frac{1}{5}\right\} ; \quad y_{j}=0, \quad j \in \mathbb{Z} \backslash\{1,2,3\},
$$

which satisfies the conditions of Theorem 6 , with $\nu=4$. Hence we may apply Algorithm 2, which then yields the sequence $\left\{p_{j}\right\} \in \ell_{0}(\mathbb{Z})$ given by

$$
\left\{p_{0}, p_{1}, p_{2}, p_{3}, p_{4}\right\}=\left\{\frac{1}{6}, \frac{3}{6}, \frac{4}{6}, \frac{3}{6}, \frac{1}{6}\right\} ; \quad p_{j}=0, \quad j \in \mathbb{Z} \backslash\{0, \ldots, 4\} .
$$

According to (6.21), the sequence $\left\{p_{j}\right\}$ satisfies the support property (1.13), with $\nu=4$, as well as the sum rule and the positivity condition (6.16), and it follows that the subdivision operator $\mathcal{S}_{\mathbf{p}}$ provides a convergent subdivision scheme. We may therefore apply Theorem 7 to deduce that the limit function $\phi$ corresponding to $\mathcal{S}_{\mathbf{p}}$ is a refinable function with refinement sequence $\left\{p_{j}\right\}$ given by (6.21) and satisfying $\{\phi(1), \phi(2), \phi(3)\}=\left\{\frac{1}{5}, \frac{3}{5}, \frac{1}{5}\right\}$.

Moreover, since $\phi$ satisfies both (1.1) and (1.12), we deduce, by recalling also the uniqueness statement in Theorem 6 , that $\phi$ is the only refinable function with refinement sequence $\left\{p_{j}\right\}$ satisfying the sum-rule condition of order $\geq 2$ and such that $\phi$ satisfies the condition (6.1). In fact, since (6.21), together with the second definition in (6.3), yields the polynomial

$$
P(z)=\left(\frac{1+z}{2}\right)^{2}\left(\frac{1+z+z^{2}}{3}\right),
$$

we deduce that $\left\{p_{j}\right\}$ satisfies the sum-rule condition of order $=2$.

The Hölder regularity of $\phi$ is obtained from (6.17) - 6.19), with $\nu=4, m=$ $2, n=1$, and $\left\{\tilde{p}_{0}, \tilde{p}_{1}, \tilde{p}_{2}, \tilde{p}_{3}\right\}=\left\{\frac{1}{3}, \frac{2}{3}, \frac{2}{3}, \frac{1}{3}\right\}$, according to which $\phi \in C_{0}^{1, \alpha}(\mathbb{R})$, where $\alpha:=\log _{2}\left(\frac{3}{2}\right) \approx 0.585$. 
Since also the conditions of Theorem 4 are satisfied for $m=2$ and $\nu=4$ by the corresponding polynomial $Y \in \pi_{2}$, as given according to (1.19) and (6.20) by $Y(z)=\frac{1}{5}\left(1+3 z+z^{2}\right)$, we may apply Algorithm 1 , with $m=2$ and $\nu=4$, to obtain the sequence

$\left\{w_{-4}, w_{-3}, w_{-2}, w_{-1}, w_{0}\right\}=\left\{-\frac{1}{6}, \frac{3}{6}, \frac{8}{6}, \frac{3}{6},-\frac{1}{6}\right\} ; \quad w_{j}=0, \quad j \in \mathbb{Z} \backslash\{-4, \ldots, 0\}$, which, together with (5.5), then yields the corresponding local interpolation operator $\mathcal{L}_{r}$ of Corollary 1, with polynomial exactness on $\pi_{1}$.

We proceed in Section 7 to consider the case where the sequence $\left\{y_{j}\right\}$ is chosen as normalized binomial coefficients.

\section{NoRmalized Binomial COEFFiCIENT VALUES ON $\mathbb{Z}$}

Following [8, Section 3], we let, for any $n \in \mathbb{N}$, the sequence $\left\{y_{j}\right\}=\left\{y_{n, j}\right\}=$ $\left\{y_{n, j}: j \in \mathbb{Z}\right\} \in \ell_{0}(\mathbb{Z})$ be defined by

$$
y_{n, j}:=\frac{1}{2^{n}}\left(\begin{array}{c}
n \\
j-1
\end{array}\right), \quad j \in \mathbb{Z},
$$

which, together with the first definition in (6.3), yields the polynomial

$$
Y(z)=Y_{n}(z)=\left(\frac{1+z}{2}\right)^{n} \text {. }
$$

Observe from (7.1) and (7.2) that

$$
\sum_{j} y_{n, j}=\sum_{j=1}^{n-1} y_{n, j}=Y_{n}(1)=1 .
$$

Moreover, (7.2) shows that $Y_{n}(0) \neq 0, \operatorname{deg}\left(Y_{n}\right)=n$, and the polynomial $Y_{n}$ has no symmetric zeros. By noting also (7.3), we conclude that the sequence $\left\{y_{j}\right\}=\left\{y_{n, j}\right\}$ satisfies the conditions of Theorem 6 , from which we therefore have the following result.

Theorem 8. For $n \in \mathbb{N}$, let the sequence $\left\{y_{n, j}\right\} \in \ell_{0}(\mathbb{Z})$ be given by (7.1). Then there exists precisely one sequence $\left\{p_{j}\right\}=\left\{p_{n, j}\right\}=\left\{p_{n, j}: j \in \mathbb{Z}\right\} \in \ell_{0}(\mathbb{Z})$ satisfying the support property

$$
\left.\operatorname{supp}\left\{p_{n, j}\right\} \subset[0, n+2]\right|_{\mathbb{Z}},
$$

as well as the condition (6.2) of Theorem 5 and the sum-rule condition of order at least 2. Moreover, $\left\{p_{n, j}\right\}$ satisfies the formulation

$$
\frac{1}{2} \sum_{j} p_{n, j} z^{j}=\frac{1}{2} \sum_{j=0}^{n+2} p_{n, j} z^{j}=\left(\frac{1+z}{2}\right)^{2} \tilde{H}_{n}(z),
$$

with $\tilde{H}_{n} \in \pi_{n}$ denoting the unique polynomial in $\pi_{n+1}$ satisfying the identity

$$
\left(\frac{1+z}{2}\right)^{n+2} \tilde{H}_{n}(z)-\left(\frac{1-z}{2}\right)^{n+2} \tilde{H}_{n}(-z)=z\left(\frac{1+z^{2}}{2}\right)^{n}, \quad z \in \mathbb{C} .
$$

For any fixed $n \in \mathbb{N}$, the polynomial $\tilde{H}=\tilde{H}_{n}$ of Theorem 8 may now be obtained from Algorithm 2 and thereby yielding the sequence $\left\{p_{n, j}\right\}$ by means of (7.5). A more efficient algorithm for the calculation of $\left\{p_{n, j}\right\}$ is obtained from the recursion 
formula in the following result, which we derive here by means of a more deductive method than the one used in [8, Section 3].

Theorem 9. For $n \in \mathbb{N}$, the sequence $\left\{p_{n, j}\right\} \in \ell_{0}(\mathbb{Z})$ in Theorem 8 satisfies the formulation

$$
\frac{1}{2} \sum_{j} p_{n, j} z^{j}=\frac{1}{2} \sum_{j=0}^{n+2} p_{n, j} z^{j}=P_{n}(z)
$$

with

$$
P_{n}(z):=\left\{\begin{array}{ll}
\left(\frac{1+z}{2}\right)^{3} Q_{k}(z), & \text { if } n=2 k-1, \\
\left(\frac{1+z}{2}\right)^{2}\left(\frac{1+z^{2}}{2}\right) Q_{k}(z), & \text { if } n=2 k,
\end{array} \quad k=1,2, \ldots,\right.
$$

where the polynomials $\left\{Q_{k}: k=1,2, \ldots\right\}$, with

$$
Q_{k} \in \pi_{2 k-2}, \quad k=1,2, \ldots,
$$

are defined recursively by

$$
\left.\begin{array}{c}
Q_{1}(z):=1 ; \\
Q_{k+1}(z):=\frac{\left(\frac{1+z^{2}}{2}\right)^{2} Q_{k}(z)-Q_{k}(-1)\left(\frac{1-z}{2}\right)^{2 k+2}}{[(1+z) / 2]^{2}}, \quad k=1,2, \ldots
\end{array}\right\}
$$

Proof. Let $n \in \mathbb{N}$. Our first step is to prove that the polynomial $\tilde{H}_{n}$ of Theorem 8 is a symmetric polynomial, in the sense that

$$
z^{n} \tilde{H}_{n}\left(z^{-1}\right)=\tilde{H}_{n}(z), \quad z \in \mathbb{C} \backslash\{0\} .
$$

To this end, we first replace $z$ by $z^{-1}$ in the identity (7.6) to obtain

$$
\left(\frac{1+z}{2}\right)^{n+2} H_{n}^{*}(z)-\left(\frac{1-z}{2}\right)^{n+2} H_{n}^{*}(-z)=z\left(\frac{1+z^{2}}{2}\right)^{n}, \quad z \in \mathbb{C} \backslash\{0\},
$$

where $H_{n}^{*}(z):=z^{n} \tilde{H}_{n}\left(z^{-1}\right), z \in \mathbb{C} \backslash\{0\}$. Since $\tilde{H}_{n} \in \pi_{n}$, it follows that $H_{n}^{*} \in \pi_{n}$. As stated in Theorem $8, \tilde{H}_{n}$ is the unique polynomial in $\pi_{n+1}$ satisfying the identity (17.6), from which we deduce that $H_{n}^{*}=\tilde{H}_{n}$, which then yields the desired symmetry result (7.11).

By setting $z=-1$ in (7.11), we obtain

$$
\tilde{H}_{n}(-1)=0, \quad \text { if } n \text { is odd. }
$$

It follows from (17.13), together with the fact that $\tilde{H}_{n} \in \pi_{n}$, that

$$
\tilde{H}_{2 k-1}(z)=\left(\frac{1+z}{2}\right) Q_{k}(z), \quad k=1,2, \ldots,
$$

for some polynomial sequence $\left\{Q_{k}: k=1,2, \ldots\right\}$ satisfying (7.9). 
Now observe from (7.6) that, for $n \in \mathbb{N}$ and any $z \in \mathbb{C}$,

$$
\begin{aligned}
& \left(\frac{1+z}{2}\right)^{n+2}\left[\left(\frac{1+z}{2}\right) \tilde{H}_{n+1}(z)-\left(\frac{1+z^{2}}{2}\right) \tilde{H}_{n}(z)\right] \\
& =\left(\frac{1-z}{2}\right)^{n+2}\left[\left(\frac{1-z}{2}\right) \tilde{H}_{n+1}(-z)-\left(\frac{1+z^{2}}{2}\right) \tilde{H}_{n}(-z)\right]
\end{aligned}
$$

from which, together with $\tilde{H}_{n} \in \pi_{n}$, we deduce that

$$
\left(\frac{1+z}{2}\right) \tilde{H}_{n+1}(z)-\left(\frac{1+z^{2}}{2}\right) \tilde{H}_{n}(z)=K\left(\frac{1-z}{2}\right)^{n+2}, \quad z \in \mathbb{C},
$$

for some constant $K$. By setting $z=-1$ in (7.15), we obtain $K=-\tilde{H}_{n}(-1)$, which we now substitute into (7.15) to obtain the identity

$$
\left(\frac{1+z}{2}\right) \tilde{H}_{n+1}(z)=\left(\frac{1+z^{2}}{2}\right) \tilde{H}_{n}(z)-\tilde{H}_{n}(-1)\left(\frac{1-z}{2}\right)^{n+2}, \quad z \in \mathbb{C}
$$

If $n=2 k-1$ for an integer $k \in \mathbb{N}$, we may now use (7.14) and (7.13) to deduce from (7.16) that

$$
\tilde{H}_{2 k}(z)=\left(\frac{1+z^{2}}{2}\right) Q_{k}(z), \quad k=1,2, \ldots
$$

If $n=2 k$ for an integer $k \in \mathbb{N}$, we next use (7.17) and (7.14) to deduce from (7.16) that

$$
\left(\frac{1+z}{2}\right)^{2} Q_{k+1}(z)=\left(\frac{1+z^{2}}{2}\right)^{2} Q_{k}(z)-Q_{k}(-1)\left(\frac{1-z}{2}\right)^{2 k+2}, \quad z \in \mathbb{C}
$$

It follows that the unique polynomial $\tilde{H}_{1} \in \pi_{1}$ satisfying the identity (7.6) for $n=1$ is given by $\tilde{H}_{1}(z)=\frac{1+z}{2}$. Hence, from (7.14), we have $Q_{1}(z)=1, z \in \mathbb{C}$.

Our proof is completed by applying (7.5), (7.14) and (7.17).

By applying the recursive formulation (7.10) in Theorem 9, we obtain the polynomials

$$
\begin{aligned}
Q_{2}(z) & =\frac{1}{4}\left(3-2 z+3 z^{2}\right) \\
Q_{3}(z) & =\frac{1}{8}\left(5-8 z+14 z^{2}-8 z^{3}+5 z^{4}\right) \\
Q_{4}(z) & =\frac{1}{64}\left(35-94 z+205 z^{2}-228 z^{3}+205 z^{4}-94 z^{5}+35 z^{6}\right),
\end{aligned}
$$


and thus, by using also (7.7) and (7.8), we calculate the sequences

$$
\begin{aligned}
& \left\{p_{1,0}, \ldots, p_{1,3}\right\}=\left\{\frac{1}{4}, \frac{3}{4}, \frac{3}{4}, \frac{1}{4}\right\} \\
& \left\{p_{2,0}, \ldots, p_{2,4}\right\}=\left\{\frac{1}{4}, \frac{2}{4}, \frac{2}{4}, \frac{2}{4}, \frac{1}{4}\right\}
\end{aligned}
$$$$
\left\{p_{3,0}, \ldots, p_{3,5}\right\}=\left\{\frac{3}{16}, \frac{7}{16}, \frac{6}{16}, \frac{6}{16}, \frac{7}{16}, \frac{3}{16}\right\}
$$$$
\left\{p_{4,0}, \ldots, p_{4,6}\right\}=\left\{\frac{3}{16}, \frac{4}{16}, \frac{5}{16}, \frac{8}{16}, \frac{5}{16}, \frac{4}{16}, \frac{3}{16}\right\}
$$$$
\left\{p_{5,0}, \ldots, p_{5,7}\right\}=\left\{\frac{5}{32}, \frac{7}{32}, \frac{5}{32}, \frac{15}{32}, \frac{15}{32}, \frac{5}{32}, \frac{7}{32}, \frac{5}{32}\right\}
$$$$
\left\{p_{6,0}, \ldots, p_{6,8}\right\}=\left\{\frac{5}{32}, \frac{2}{32}, \frac{8}{32}, \frac{14}{32}, \frac{6}{32}, \frac{14}{32}, \frac{8}{32}, \frac{2}{32}, \frac{5}{32}\right\}
$$$$
\left\{p_{7,0}, \ldots, p_{7,9}\right\}=\left\{\frac{35}{256}, \frac{11}{256}, \frac{28}{256}, \frac{140}{256}, \frac{42}{256}, \frac{42}{256}, \frac{140}{256}, \frac{28}{256}, \frac{11}{256}, \frac{35}{256}\right\}
$$$$
(7.26)\left\{p_{8,0}, \ldots, p_{8,10}\right\}=\left\{\frac{35}{256},-\frac{24}{256}, \frac{87}{256}, \frac{64}{256}, \frac{6}{256}, \frac{176}{256}, \frac{6}{256}, \frac{64}{256}, \frac{87}{256},-\frac{24}{256}, \frac{35}{256}\right\} \text {, }
$$

which agree with the numerical results in [8, Section 3].

Observe that, for $n=1, \ldots, 7$, we have $p_{n, j}>0, j=0, \ldots, n+2$, with also $Q_{j}(-1) \neq 0, j=1, \ldots, 4$.

Hence we may now deduce the following result from Theorems 9, 8, 7 and 6 .

Corollary 2. For any integer $n \in\{1, \ldots, 7\}$, there exists precisely one refinable function $\phi_{n}$, with refinement sequence $\left\{p_{n, j}\right\}$ given in (7.19) - (7.25) such that

$$
\phi_{n}(j)=\frac{1}{2^{n}}\left(\begin{array}{c}
n \\
j-1
\end{array}\right), \quad j \in \mathbb{Z},
$$

and with $\left\{p_{n, j}\right\}$ satisfying the sum-rule condition of order $m$, where

$$
m= \begin{cases}3, & \text { if } n \text { is odd } \\ 2, & \text { if } n \text { is even } .\end{cases}
$$

Observe from (7.2) that $Y_{1}=E_{3}$, the Euler-Frobenius polynomial corresponding to the quadratic cardinal $B$-spline $N_{3}$, as obtained in Example A, so that we may deduce from Corollary 2 that $\phi_{1}=N_{3}$.

For $n=2, \ldots, 7$, we may apply (6.17) - 6.19) to derive the Hölder regularity results in Table 1 for the refinable function $\phi_{n}$ of Corollary 2 .

Since also $\phi_{1}=N_{3} \in C_{0}^{1,1}(\mathbb{R}) \backslash C^{2}(\mathbb{R})$, we deduce from Table 1 that the best smoothness result is obtained for $n=3$, with $\phi_{3} \in C_{0}^{2, \log _{2}\left(\frac{4}{3}\right)}(\mathbb{R})$.

Finally, observe that, for any $n \in\{1, \ldots, 7\}$, the polynomial $Y=Y_{n}$ in (7.2) satisfies the conditions of Corollary 1, with $m$ given as in (7.28), and $\nu=n+2$, according to which the polynomial identity (3.17) is then given by

$$
\left(\frac{1+z}{2}\right)^{2\left\lfloor\frac{1}{2}(n+3)\right\rfloor} \tilde{X}(z)-\left(\frac{1-z}{2}\right)^{2\left\lfloor\frac{1}{2}(n+3)\right\rfloor} \tilde{X}(-z)=z^{2\left\lfloor\frac{1}{2}(n+3)\right\rfloor-1}, \quad z \in \mathbb{C} .
$$


TABLE 1. Hölder regularity of the refinable functions $\phi_{2}, \ldots, \phi_{7}$ of Corollary 2.

\begin{tabular}{|l|l|l|l|l|l|l|}
\hline \multicolumn{7}{|c|}{$\phi_{n} \in C_{0}^{k, \alpha}(\mathbb{R})$} \\
\hline$n$ & $k$ & $\alpha$ & & $n$ & $k$ & $\alpha$ \\
\hline 2 & 1 & 1 & 5 & 1 & $\log _{2}\left(\frac{8}{7}\right) \approx 0.1926$ \\
3 & 2 & $\log _{2}\left(\frac{4}{3}\right) \approx 0.4150$ & 6 & 0 & $\log _{2}\left(\frac{16}{15}\right) \approx 0.0931$ \\
4 & 1 & $\log _{2}\left(\frac{8}{7}\right) \approx 0.1926$ & 7 & 0 & $\log _{2}\left(\frac{256}{245}\right) \approx 0.0634$ \\
\hline
\end{tabular}

To obtain the solution

$$
\tilde{X} \in\left\{\begin{array}{lll}
\pi_{n+1}, & \text { if } & n \text { is odd; } \\
\pi_{n}, & \text { if } & n \text { is even }
\end{array}\right.
$$

of the identity (7.29), we may therefore apply the formula (4.15) directly to deduce from (3.17) in Corollary 1 that, with the sequence $\left\{w_{n, j}\right\} \in \ell_{0}(\mathbb{Z})$ defined by

$$
\begin{array}{r}
\sum_{j} w_{n, j} z^{j}:=2 z^{-\left\lfloor\frac{1}{2}(n+3)\right\rfloor-1}\left(\frac{1+z}{2}\right)^{m} \sum_{j=0}^{\left\lfloor\frac{1}{2}(n+3)\right\rfloor-1}\left(\begin{array}{c}
\left\lfloor\frac{1}{2}(n+3)\right\rfloor+j-1 \\
j
\end{array}\right) \\
\times\left[\frac{1}{2}\left(1-\frac{z+z^{-1}}{2}\right)\right]^{j},
\end{array}
$$

where the integer $m$ is given by (7.28), the corresponding approximation operator $\mathcal{L}_{r}=\mathcal{L}_{n, r}$, as given by (5.5), with $\left\{w_{j}\right\}=\left\{w_{n, j}\right\}$ and $\phi=\phi_{n}$, is a local interpolation operator as in (A) and (B) of Section 1, with polynomial exactness on $\pi_{m-1}$.

For $n=1$, as is evident from the fact that $\phi_{1}=N_{3}$, the sequence $\left\{w_{j}\right\}=\left\{w_{1, j}\right.$ : $j \in \mathbb{Z}\}$ in (7.31) is given by (5.14).

For $n=2$ and $n=3$, we calculate by means of (7.31) the sequences

$$
\begin{gathered}
\left\{w_{2,-4}, w_{2,-3}, w_{2,-2}, w_{2,-1}, w_{2,0}\right\}=\left\{-\frac{1}{4}, \frac{2}{4}, \frac{6}{4}, \frac{2}{4},-\frac{1}{4}\right\} \\
w_{2, j}=0, \quad j \in \mathbb{Z} \backslash\{-4, \ldots, 0\}
\end{gathered}
$$

and

$$
\left.\begin{array}{c}
\left\{w_{3,-6}, w_{3,-5}, w_{3,-4}, w_{3,-3}, w_{3,-2}, w_{3,-1}, w_{3,0}, w_{3,1}\right\} \\
=\left\{\frac{3}{32},-\frac{9}{32},-\frac{7}{32}, \frac{45}{32}, \frac{45}{32},-\frac{7}{32},-\frac{9}{32}, \frac{3}{32}\right\} ; \\
w_{3, j}=0, \quad j \in \mathbb{Z} \backslash\{-6, \ldots, 1\},
\end{array}\right\}
$$

which, together with (5.5), yield local interpolation operators $\mathcal{L}_{2, r}$ and $\mathcal{L}_{3, r}$, with polynomial exactness on, respectively, $\pi_{2}$ and $\pi_{3}$. 


\section{REFERENCES}

[1] A. S. Cavaretta, W. Dahmen, and C. A. Micchelli, Stationary subdivision, Mem. Amer. Math. Soc. 93 (1991), no. 453, vi+186, DOI 10.1090/memo/0453. MR 1079033(92h:65017)

[2] C. Chui and J. de Villiers, Wavelet Subdivision Methods, GEMS for rendering curves and surfaces; with a foreword by Tom Lyche, CRC Press, Boca Raton, FL, 2011. MR2683008 $(2011 \mathrm{k}: 42001)$

[3] C. K. Chui, An Introduction to Wavelets, Wavelet Analysis and its Applications, vol. 1, Academic Press, Inc., Boston, MA, 1992. MR1150048 (93f:42055)

[4] C. K. Chui and J. M. De Villiers, Applications of optimally local interpolation to interpolatory approximants and compactly supported wavelets, Math. Comp. 65 (1996), no. 213, 99-114, DOI 10.1090/S0025-5718-96-00672-2. MR1322886 (96d:41003)

[5] W. Dahmen, T. N. T. Goodman, and C. A. Micchelli, Compactly supported fundamental functions for spline interpolation, Numer. Math. 52 (1988), no. 6, 639-664, DOI 10.1007/BF01395816. MR946381 (89i:65011)

[6] I. Daubechies, Orthonormal bases of compactly supported wavelets, Comm. Pure Appl. Math. 41 (1988), no. 7, 909-996, DOI 10.1002/cpa.3160410705. MR951745 (90m:42039)

[7] I. Daubechies, Ten Lectures on Wavelets, CBMS-NSF Regional Conference Series in Applied Mathematics, vol. 61, Society for Industrial and Applied Mathematics (SIAM), Philadelphia, PA, 1992. MR 1162107 (93e:42045)

[8] J. M. de Villiers, C. A. Micchelli, and T. Sauer, Building refinable functions from their values at integers, Calcolo 37 (2000), no. 3, 139-158, DOI 10.1007/s100920070006. MR 1790328 (2002g:65179)

[9] J. M. De Villiers and C. H. Rohwer, Optimal local spline interpolants, Special issue on the 11th South African symposium on numerical mathematics (Umhlanga, 1985), J. Comput. Appl. Math. 18 (1987), no. 1, 107-119, DOI 10.1016/0377-0427(87)90059-8. MR891413(88i:41017)

[10] C. A. Micchelli, Mathematical Aspects of Geometric Modeling, CBMS-NSF Regional Conference Series in Applied Mathematics, vol. 65, Society for Industrial and Applied Mathematics (SIAM), Philadelphia, PA, 1995. MR1308048 (95i:65036)

[11] C. A. Micchelli and Q. Sun, Refinable functions from their values at integers, Vietnam J. Math. 30 (2002), no. 4, 395-411. MR1949937 (2003m:42062)

[12] I. J. Schoenberg, Cardinal Spline Interpolation, Conference Board of the Mathematical Sciences Regional Conference Series in Applied Mathematics, No. 12, Society for Industrial and Applied Mathematics, Philadelphia, Pa., 1973. MR0420078 (54 \#8095)

Department of Mathematical Sciences, Mathematics Division, Stellenbosch UniverSity, South Africa - And - African Institute for Mathematical Sciences (AIMS), Muizenberg, South Africa

Department of Mathematical Sciences, Mathematics Division, Stellenbosch UniverSity, South Africa - and - African Institute for Mathematical Sciences (AIMS), Muizenberg, South Africa 